\title{
Theory of Aharonov-Bohm oscillations resulting from a charge density wave condensate flowing across an array of columnar defects in magnetic field
}

\author{
Alexander S. Rozhavsky* \\ Centre de Recherches sur les Tres Basses Temperatures, laboratoire associe a l'Universite Joseph Fourier, \\ C. N. R. S., BP 166, 38042 Grenoble-Cedex 9, France
}

Received March 30, 1998; revised May 19, 1998

\begin{abstract}
We propose a microscopic theory for the Aharonov-Bohm oscillations, observed by Latyshev et al. in the magnetotransport experiment in $\mathrm{NbSe}_{3}$ with a charge density wave (CDW). CDW slides across an array of columnar defects in a high magnetic field. For the charge carrying quanta of CDW these defects are elementary solenoids carrying a magnetic flux. The quantum CDW current acquires a component oscillating with the flux, with the period $h c / 2 e$. Its magnitude is proportional to the concentration of columnar defects. The lower limit to the phase breaking length for these oscillations is set by the minimal Lee-Rice coherence length.
\end{abstract}

PACS: 71.45.Lr, 72.15.-v

The sliding charge density and spin-density waves (CDW and SDW) in quasi-one-dimensional metals represent the amazing examples, apart from the superconductivity, of collective transport by a moving quantum ground state at high enough temperatures.

The most striking feature is the existence of a nonlinear dc-current along the high conducting direction produced by the Fröhlich collective mode (see the review papers [1]). The Fröhlich conductivity emerges in electric fields exceeding the threshold value $E_{T}\left(E>E_{T}\right)$. Until recently, all the $\mathrm{CDW}$-transport experiments had been more or less successfully explained by the theories which had considered CDW in a classical manner: either phenomenologically as a rigid object moving in a periodic potential, or microscopically, as a deformable medium in which the topologically stable domain walls (the CDW solitons) serve as the elementary CDW-charge carriers (see [1]).

The pioneer experiment which had evidently shown the quantum nature of the $\mathrm{CDW}$-solitons was performed by Latyshev et al., [2]. A thin film of a CDW-conductor $\mathrm{NbSe}_{3}$ was irradiated by heavy ions of Xe. The Xe-ions produced an array of identical parallel tracks [columnar defects (CD)] piercing the film, and the host lattice inside the $\mathrm{CD}$ was destroyed. The radius $R$ of each $\mathrm{CD}$ is of the order of $100 \AA$. The irradiated film was placed in a strong magnetic field $H \approx 20 \mathrm{~T}$ (Fig. 1) and the nonlinear conductance was measured. In electric fields above the threshold, $E>E_{T}$, the transport current appeared to be an oscillatory function of the magnetic field with the period of oscillation

$$
\delta H \cong \frac{h c}{2 e \pi R^{2}} .
$$

The relative magnitude of the oscillatory component of the transport current is of the order of $0,25 \%$.

The oscillatory dependence on $H$ clearly indicates the Aharonov-Bohm effect (ABE) nature of the observed phenomenon, the period of the ABEoscillations with the magnetic flux being $\Delta \Phi=h c / 2 e$.

* Permanent address: B. I. Verkin Institute for Low Temperature Physics and Engineering, 47 Lenin Ave., 310164 Kharkov, Ukraine 


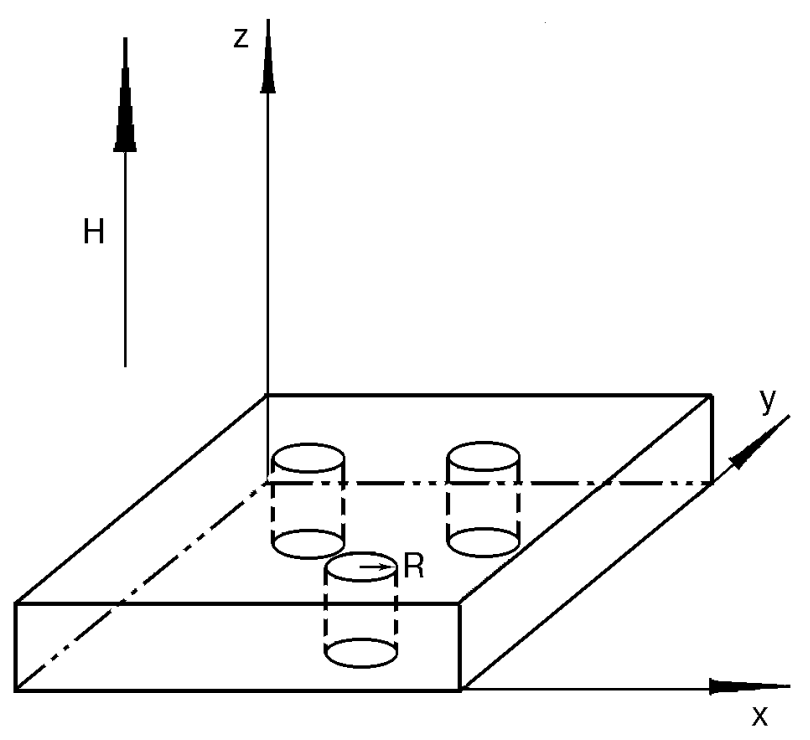

I, E

Fig. 1. Geometry of the experiment [2]. Magnetic field $\mathbf{H}$ is oriented along the axis of the columnar defects of radius $R$ ( $z$ axis), the transport current I flows along the electric field $\mathbf{E}$ ( $x$ axis).

The striking feature of this $\mathrm{ABE}$ is that it is observed at such high temperatures $(T \approx 50 \mathrm{~K})$ when single-electron $\mathrm{ABE}$ is totally suppressed by the electron-phonon scattering. Therefore, it was assumed in [5] that those oscillations can be attributed to the quantum $\mathrm{CDW}$-solitons which encircle the CDs pierced by the magnetic field thus affecting the collective Fröhlich current. In other words, the CDs serve as the elementary solenoids for quantum solitons.

To summarize, the experiment [2] is really the first evidence for a high-temperature quantum coherent effect in a non-superconducting material. It brings us to a qualitatively new level in understanding the nature of a CDW current state: it demonstrates unambiguously the quantum origin of a collective current which was masked in all the previous studies. It demands therefore a new theoretical description of transport phenomena in CDW-conductors.

A theory that aims to describe the ABE in CDW transport should include the quantum description of the CDW charge carriers and should explain the anomalously large CDW phase breaking length $L_{\varphi}^{C D W}$, strongly exceeding the one for a single electron transport at such temperatures, which makes $\mathrm{ABE}$ observable at high temperatures.

In this paper we develop such a theory, using the concepts, formulated in [3,4], where the idea of the CDW charge carriers quantization has been put forward and the persistent current of the CDW-condensate has been studied for the first time.

It is well known, that the $\mathrm{ABE}$ in conductors can be observed in two ways: (i) as oscillations of a diamagnetic moment (of persistent currents) in isolated loops, and (ii) as oscillations of conductance in open circuits containing loops. As the ABE is intimately connected to the topological structure of a charge carrier wave function in a multiply connected conductor, the basic period of oscillations with flux in both those schemes is the same.

The qualitative theory of persistent currents in CDW in the idealized model of a single-chain CDW loop was first proposed by Krive and the author [3] and was elaborated later on in a series of papers [4]. It was shown there that the persistent current in CDW is formed by the topologically non-trivial CDW-excitations, viz, solitons and instantons. The basic period of the persistent current oscillations with magnetic flux is found to be $\Phi_{S}=h c / 2 e$, just as in the experiment [2]. Although the measurements of a persistent current in CDW are beyond the present technology facilities, the understanding of the role of the topological CDW-excitations in the $\mathrm{ABE}$ is essential for solution of the quantum transport problem. The qualitative picture of the AB-oscillations in the geometry of interest was first discussed by the author [5].

The paper is organized as follows.

In the first chapter, we give a proper method of CDW quantization and formulate an exactly solvable model of a quantum CDW transport along a single chain with a loop pierced by a magnetic flux. Such a loop mimics a columnar defect.

In the second chapter, we propose a model of a columnar defect in a CDW-material. In the third part, we show that the long range $\mathrm{CDW}$-coherence allows to reduce the problem of a transport current through a dilute array of CDs to the one studied in the first chapter, and calculate the transport current oscillating with flux. The relative magnitude of the oscillatory current estimated along our formulas for the parameters of the experiment [2] is of the order of $10^{-2}-10^{-3}$, which is in a good agreement with the experimental data.

\section{Mathematical formalism. Aharonov-Bohm oscillations of a CDW transport current in a toy $1 D$ model}

The Peierls-Fröhlich order parameter is $\Delta \exp (i \varphi)$ where $\Delta$ is the gap in a single electron spectrum and the gradients of $\varphi$ define, via the Fröhlich relations [1], the collective CDW current, 
$j$, and the charge density fluctuations, $\rho$, in a single chain:

$$
\begin{gathered}
j=-\frac{e}{\pi} \frac{\partial \varphi}{\partial t}, \\
\rho=\frac{e}{\pi} \frac{\partial \varphi}{\partial x},
\end{gathered}
$$

where $e$ is the electron charge, and $x$ denotes the coordinate along the chain.

Note that the Eqs. (1) have, at first glance, an incorrect vector dimensionality. Indeed, the Eq. (1a) comprises a scalar operator that acts on a scalar to give a vector quantity, and the Eq. (1b) comprises the vector operator acting on a scalar to give a scalar. It is necessarily the case that there is an implicit direction implied. Such a direction is the one along the chains. The phase $\varphi$ (the CDW variable), strictly speaking, is a quantum Bose field. This can easily be seen in the path integral formulation of the Peierls-Fröhlich problem (see, e.g., the review by Krive et al. in Ref. 1). Accordingly, the Eqs. (1) are the operator equations. The measured current is:

$$
j_{t}=-\frac{e}{\pi}\left\langle\frac{\partial \varphi}{\partial t}\right\rangle=j_{c}+j_{q},
$$

where the brackets denote quantum averaging. In electric field above the threshold, $E>E_{T}$, the transport current (2) has two components: the classic $j_{c}$ and the quantum, $j_{q}$. The former is produced by the mean component of the order parameter, and the latter by its fluctuations. The theory of a classic CDW dc-transport is well developed (see [1]). For our purposes we must find the quantum dc-current $j_{q}$ expressed in terms of wavefunctions of individual $\mathrm{CDW}$-charge carriers. In the Aharonov-Bohm geometry, the wavefunctions are sensitive to magnetic flux variations providing oscillations of a transport current. To quantize CDW, we use the Bose-Fermi duality transformations in $1 D$ [6]. This powerful tool allows us to map the results of the electron theory of metals and semiconductors onto quantum CDW. In particular, quantum solitons of CDW, which serve as elementary charge carriers, turn out to be equivalent to spinless conduction electrons in a $1 D$ semiconductor (see, e.g., [7]). Evidently, the oscillatory ABEs, existing in mesoscopic conductors, have their analogs in CDW.

The dual transformations for the normally ordered operators are (see, e.g., [7]):

$$
\frac{1}{\sqrt{\pi}} \partial_{t} \varphi \rightleftarrows \bar{\psi} \sigma_{x} \psi
$$

$$
\frac{1}{\sqrt{\pi}} \partial_{x} \varphi \rightleftarrows-\bar{\psi} \sigma_{y} \psi
$$

where $\psi$ is a two-component Dirac spinor, $\bar{\psi}=\psi^{+} \sigma_{y}, \sigma_{\mu}$ are the Pauli matrices.

The qualitative picture underlying the transformations (3) is that the statistics in $1 D$ is ill defined. One cannot merely place two solitons at one point which, roughly speaking, allows us to treat them as fermions. The Eqs. (3) introduce explicitly the desired description of a CDW in terms of the wavefunctions of the CDW charge carriers (solitons and antisolitons).

To show how this scheme works, consider the AB-oscillations of $j_{q}$ in a single chain with a loop, of a perimeter $L$, pierced by a magnetic flux $\Phi$ (Fig. 2). Consider for simplicity an incommensurate CDW described by Lagrangians:

$$
\mathcal{L}=N_{0}\left\{\frac{1}{2}\left(\partial_{t} \varphi\right)^{2}-\frac{c_{0}^{2}}{2}\left(\partial_{x} \varphi\right)^{2}\right\}
$$

in linear chains connected to the loop, and

$$
\mathcal{L}=N_{0}\left\{\frac{1}{2}\left(\partial_{t} \varphi\right)^{2}-\frac{2 \pi^{2} c_{0}^{2}}{L^{2}}\left(\partial_{\theta} \varphi\right)^{2}\right\}-\frac{e \Phi}{\pi c L}\left(\partial_{t} \varphi\right)
$$

in a loop.

Here $N_{0}=\hbar / \alpha^{2} v_{F}, \alpha=c_{0} / v_{F}<1$ is the parameter of adiabaticity in the Peierls-Fröhlich theory, $c_{0}$ is the phase velocity of CDW.

The Lagrangian (4a) is well known in the theory of CDW. The Lagrangian (4b) contains the topological term which describes coupling of a Fröhlich current (1a) to a vector potential $A=\Phi / L$ in the loop:

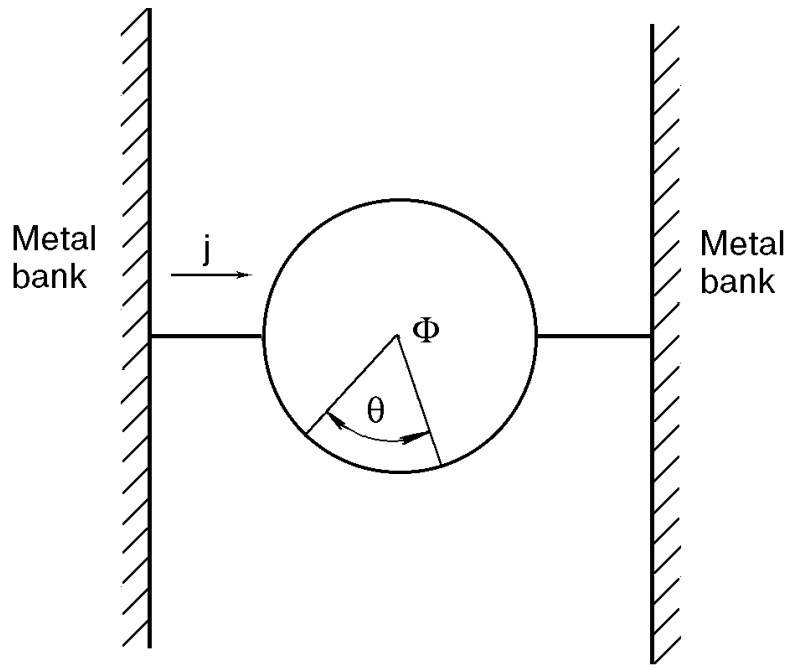

Fig. 2. Schematic view of a CDW-transport along a single chain containing a loop with a perimeter $L$, pierced by the magnetic flux $\Phi ; j$ is the transport current density; $\theta$ is the angle variable. 


$$
\mathcal{L}_{\text {int }}=\frac{j A}{c} .
$$

The topological term in (4b) was introduced for the first time in the paper by Bogachek et al., Ref. 4. On a microscopic level, it arises nonperturbatively due to the chiral anomaly phenomenon in a $1 D \mathrm{CDW}[7,8]$. The doubling of the electron charge in (4b) is the result of a summation over spin projections of electrons out of which the CDW is formed. The effective charge $2 e$ leads to the oscillations of the persistent and transport currents with flux with the period $\Phi_{s}=h c / 2 e[4,5]$.

To obtain the $j_{q}$ one has to find the transparency of the loop. It is highly unacceptable to do it in terms of $\varphi$-field using for example the instanton approach [4]. It is more convenient to use the dual fermion language in which this problem can be reduced to the one already solved in Ref. 9. Indeed, the Lagrangians (4a), (4b) take the Dirac form:

$$
\mathcal{L}=N_{0} i \bar{\psi} \gamma_{\mu} \partial_{\mu} \psi
$$

and

$$
\mathcal{L}=N_{0} i \bar{\psi} \gamma_{\mu}\left(\partial_{\mu}-i \frac{e}{\pi c L} \Phi \delta_{\mu, x}\right) \psi,
$$

where $\gamma_{\mu}=\left(\sigma_{y}, c_{0} \sigma_{x}\right), \mu=(t, x)$.

The scattering problem of interest has been solved in Ref. 9 for a nonrelativistic electron. One can easily show that the results obtained in Ref. 9 remain valid in our case.

The CDW quantum transport current takes the form [10]:

$$
j_{q}(\Phi)=\frac{2 e}{h} \int d \varepsilon g(\varepsilon)\left[f_{L}(\varepsilon)-f_{R}(\varepsilon)\right]
$$

The integration is performed over the energy $\varepsilon$. Here $g(\varepsilon)$ is the transmission probability, which depends explicitly on the CDW wave functions, $f_{R, L}$ are the distribution functions of the left and right moving dual fermions. For simplicity, we assume that the relaxation occurs in leads, which means that

$$
f_{R, L}=f_{0}\left(\varepsilon \pm p v_{D}\right)
$$

where $f_{0}$ is the Fermi function and $v_{D}$ is the drift velocity. For sliding CDW, $v_{D}$ is not zero in electric fields above the threshold $E_{T}$. It is convenient to rewrite (6) in the form:

$$
j_{q}(\Phi)=j_{C} \int d \varepsilon g(\varepsilon) \frac{\left[f_{0}\left(\varepsilon+p v_{D}\right)-f_{0}\left(\varepsilon-p v_{D}\right)\right]}{2 p v_{D}},
$$

where $j_{c}$ is the classic current at zero temperature along a chain free of a loop:

$$
j_{C}=\frac{e \tilde{p}_{F}{ }_{D}(E)}{\pi \hbar} .
$$

The Fermi energy for dual fermions is of the order of $\Delta$. This is the maximum kinetic energy which the CDW soliton (the dual fermion) possesses [11]. Correspondingly the cut-off to the Dirac theory, for dual fermions is:

$$
\tilde{p}_{F} \sim \frac{\Delta}{c_{0}} \sim \frac{\hbar}{\alpha \xi_{0}}
$$

where $\xi_{0}=\hbar v_{F} / \Delta$ is the amplitude coherence length.

The function $g(\varepsilon)$ was calculated in Ref. 9 to be:

$$
g(\varepsilon)=\frac{4 \eta^{2} \cos ^{2}\left(\pi \Phi / \Phi_{s}\right) \sin ^{2}(\pi k)}{\eta^{2} \sin ^{2}(2 \pi k)+p^{2}(k, \eta, \Phi)}
$$

where

$$
\begin{gathered}
p(k, \eta, \Phi)=b^{2} \cos \left(\frac{2 \pi \Phi}{\Phi_{s}}\right)+a^{2}-(1-\eta) \cos (2 \pi k), \\
k=\frac{\varepsilon L}{2 \pi \hbar c_{0}} .
\end{gathered}
$$

The phenomenological parameter $\eta, 0 \leq \eta \leq 1 / 2$, determines the connection between the linear chain and the loop. The coefficients $a, b$ are:

$$
a=\frac{1}{2}\left[(1-2 \eta)^{1 / 2}-1\right], \quad b=\frac{1}{2}\left[(1-2 \eta)^{1 / 2}+1\right] .
$$

The value of $\eta$ will be specified in the Ch. 2 .

Note that the Eqs. (6) and (10) are valid when the perimeter of a loop is small compared to the phase breaking length $L_{\varphi}^{C D W}$. In a sliding $1 D \mathrm{CDW}$ the lower limit to $L_{\varphi}^{C D W}$ is set by the Lee-Rice phase coherence length $l_{\|}$which is typically 1$10 \mu \mathrm{m}$ [1]. This clearly shows why the CDW $\mathrm{ABE}$ is observed at sufficiently high temperatures, when the single electron $\mathrm{ABE}$, characterized by $L_{\varphi}^{(e)} \sim 10^{3}-10^{3} \AA$, is suppressed. A three-dimensional sample of $\mathrm{NbSe}_{3}$ is characterized by three coherence lengths: $l_{\|}$along the chains in $x$-direction, $l_{\perp}$ along the $y$ direction and $l_{d}$ along the less conducting axis, $z: l_{d} \ll l_{\perp} \ll l_{\|}$, and, correspondingly, by three phase velocities $c_{z} \ll c_{y}<<c_{0}$. In an actual experiment [2], the phase breaking length is governed by the sample thickness $d$ in a less conducting direction. In this context, the oscillatory effect in 
CDW is the mesoscopic one in spite of it resemblance to the flux quantization in superconductors.

Under the conditions of the experiment [2], the $\mathrm{CD}$ hole diameter contains several tens of chains and the model has to be reformulated keeping safe two basic features: (i) a qualitative picture of the quantum transport of $\mathrm{CDW}$ along a single chain [Eqs. (6), (10)], and (ii) the long distance phase coherence in a sliding CDW.

\section{A model of a columnar defect in a CDW material}

According to [2], the $\mathrm{CD}$ is a real hole with a diameter 10-12 $\mathrm{nm}$ surrounded by a damaged region which adds a further $2-4 \mathrm{~nm}$ to the effective diameter.

Consider the distribution of a phase around a single cylindric CD with the radius $R$ (Fig. 3). Inside the $\mathrm{CD}$, the host material is destroyed and, accordingly, $\Delta=0$. This local defect creates Friedel oscillations of the electron charge that compete with the CDW charge modulation over an atomic distance scale $\delta_{1} \sim 10 \AA$ [12]. The suppressed $\Delta$

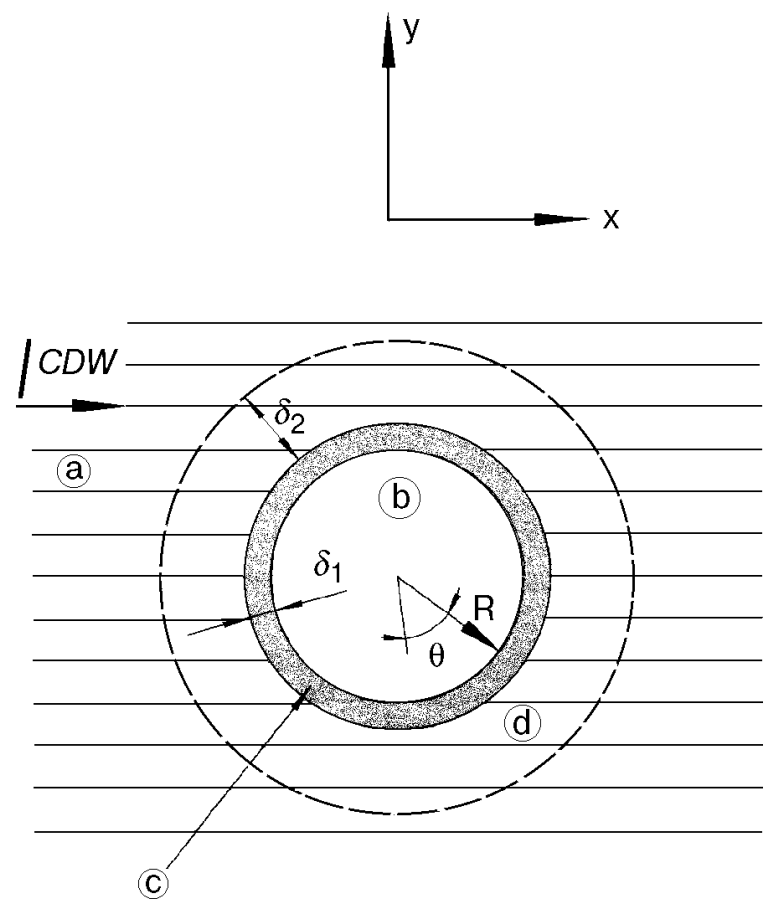

Fig. 3. The model of $\mathrm{CD}$ adopted in the theory: a) conducting chains are oriented along the $x$-axis; b) the real hole; c) the region of the edge states of the width $\sim \delta_{1}$; d) the interface layer of the width $\sim \delta_{2}$ around the CD containing large phase gradients. The high energy heavy ion produces a round hole which pierces the sample along the $z$-axis. The CD-hole is surrounded by a layer of width $\delta_{1}$, within which the phase is confined to $\varphi=\varphi_{0}$ (see the text). We assume that the positions of conducting chains are not disturbed away from the CD: $|x|>R|\cos \theta|$, $|y|>R|\sin \theta| . I^{C D W}$ is the transport current; $\theta$ is the angle variable. causes a strong phase gradients $|\partial \varphi / \partial r|>>|1|^{-1}$ localized around the CD within a strip of width $\delta_{2}$ which is of the order of several $\xi_{0}$ [12]. The potential barrier created by these phase gradients is of the order of $\Delta$ [12] (Fig. 4). It separates the phase $\varphi$ inside $\delta_{1}$ from the bulk. The local phase $\varphi$ in the region $R+\delta_{1} \propto r<<R+\delta_{2}$ is adjusted to a certain optimal value $\varphi_{0}$ that matches the Friedel oscillations. The value $\varphi_{0}$ differs from the one established in the bulk, its excitations can be regarded as the edge states. We make a plausible assumption that inside the layer $\sim \delta_{1}$ there exists random $1 D$ chain paths encircling the $\mathrm{CD}$, along which the CDW current is again given by the Eq. (1a). The CDW edge state phase velocity along those $1 D$ paths is $\bar{c}$ where $c_{y}<\bar{c}<c_{0}$. Such random chains emerge as the result of a strong damage of a host lattice caused by a heavy ion. The average phase $\varphi$ in the bulk away from the CD still remains correlated over the Lee-Rice lengths [12]. This qualitative picture is an assumption of our model.

Consider the scattering of the classic and quantum CDW excitations on a CD. In the classical picture the excitations of $\varphi_{0}$ (the edge states) are confined to a $\mathrm{CD}$ in a radial direction, they have only the azimuthal momenta and do not contribute

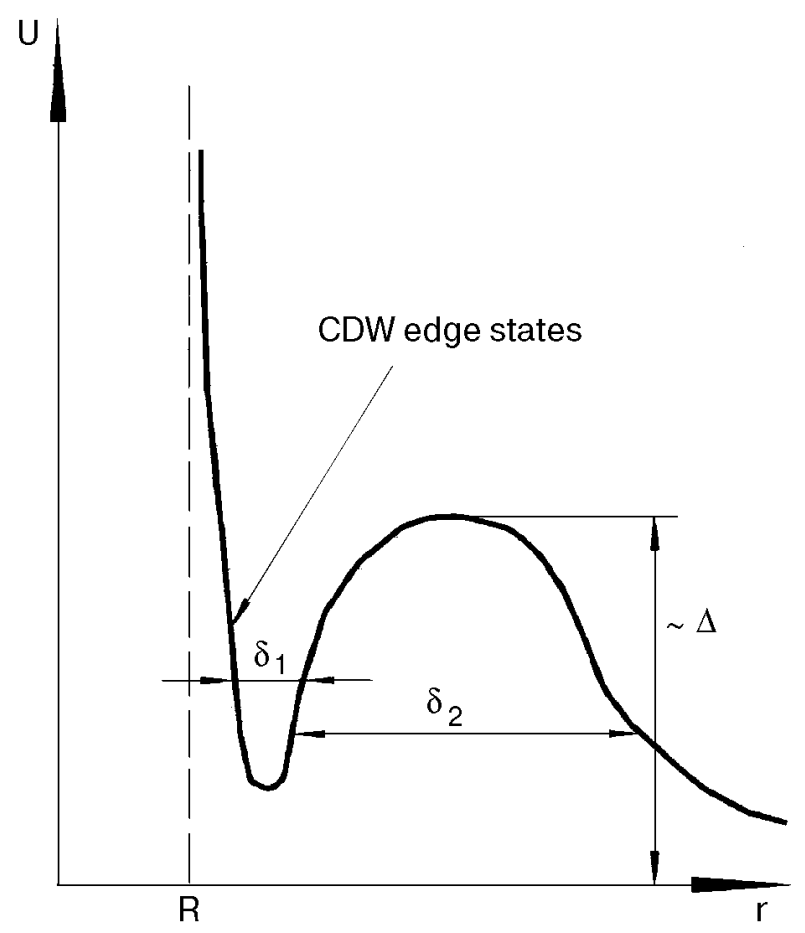

Fig. 4. The potential $U$ created by the $\mathrm{CD}$ for the CDW excitations as a function of the radial coordinate $r$. The destroyed host material occupies the region $r<R$. The potential barrier of the order of $\Delta$ produced by the large phase gradients in the region $R+\delta_{1}<r<R+\delta_{2}$ separates the edge states localized at $R<r<R+\delta_{1}$ from the bulk. $R$ is the CD radius. 

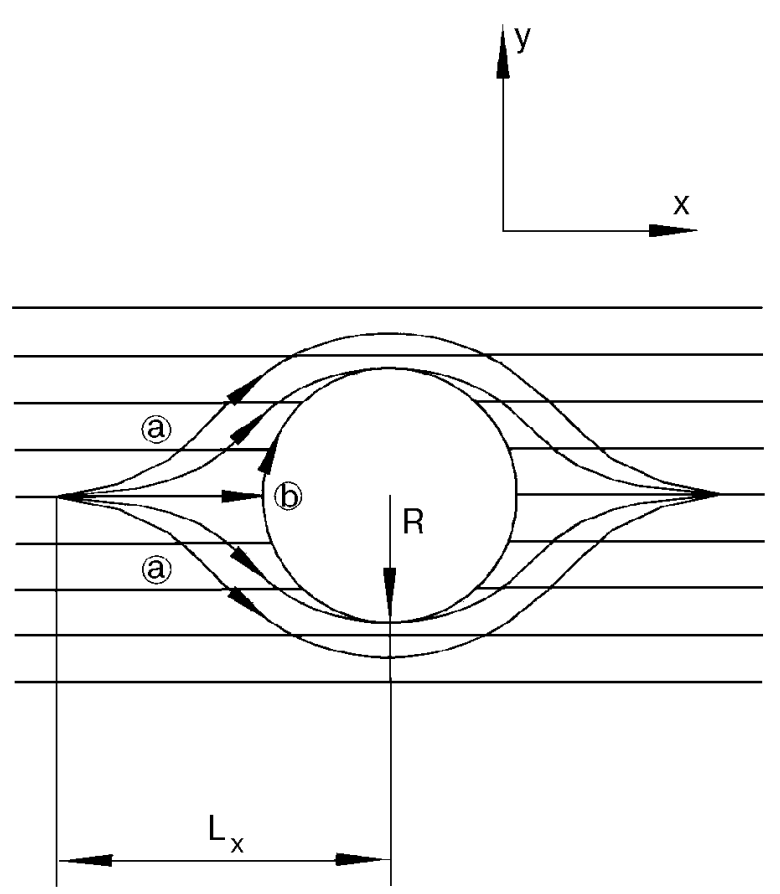

Fig. 5. The trajectories along which the classic current (a) and quantum current $(b)$ encircle the CD. $L_{x}$ is the classical current spreading length (14).

to the current flow. The classic current spreads around the $\mathrm{CD}$ in the way plotted in Fig. 5, $a$. The characteristic spreading length $L_{x}$ can be estimated from the equation of motion for a $3 D \mathrm{CDW}$ Lagrangian:

$$
\begin{aligned}
\mathcal{L}= & n_{2} N_{0} \int d x d \mathbf{r}_{\perp}\left\{\frac{1}{2}\left(\partial_{t} \varphi\right)^{2}-\frac{c_{0}^{2}}{2}\left(\partial_{x} \varphi\right)^{2}-\right. \\
& \left.-\frac{2 Z T_{c}^{2}}{\pi \hbar v_{F} N_{0}} \cos \left(\varphi_{\mathbf{r}_{\perp}}-\varphi_{\mathbf{r}_{\perp}+1}\right)\right\},
\end{aligned}
$$

where $n_{2}$ is the $2 D$ density of conducting chains, $T_{c}$ is the critical temperature of a $3 D$ phase transition, and $Z$ is the number of the nearest neighbor chains. Assuming the thickness of a sample $d<<l_{d}$ and putting $\partial x \sim 1 / L_{x}, \partial y \sim 1 / L$, we get an estimate:

$$
L_{x} \sim L \frac{c_{0}}{c_{y}} \sim L \frac{\varepsilon_{\mathrm{r}}}{T_{c}}>>L
$$

where

$$
c_{y}=\frac{\sqrt{2 Z} T_{c}}{\hbar n_{2}^{1 / 2}} \alpha \ll c_{0} .
$$

In $\mathrm{NbSe}_{3} c_{y} \approx 0.1 c_{0}$. Classic trajectories (14) contribute to the magnetoresistance but not to the $\mathrm{ABE}$.
Contrary to the classic picture, the quantum CDW-particles encircle the CD in a quite different way (Fig. 5,b). Quantum particles propagate freely along the chain away from the $\mathrm{CD}$, then penetrate under the potential barrier (Fig. 4) and mix with the edge states localized near the hole. Because the dispersion of the edge state trajectories is small as far as $\delta_{1}<<L$ and $k_{F} \delta_{1} \approx 1$, the CDW quanta encircle the CD along a path of a fixed length $L$. Quantum paths obviously contribute to the AB-oscillations.

Now we can formulate a quantum model of a CDW which contains a single CD.

Save $N_{0}$, the excitations of the phase localized in the chains away from the $\mathrm{CD}$ are described by the Lagrangian:

$$
\mathcal{L}=\sum_{n} i \bar{\psi}_{n} \gamma_{\mu} \partial_{\mu} \psi_{n}
$$

where $n$ labels the chain. There are $N_{\Phi}$ chains which connect the $\mathrm{CD}$ :

$$
N_{\Phi} \approx \frac{L d}{4 \pi} n_{2}
$$

The edge excitations of $\varphi_{0}$ are described by the Lagrangian:

$$
\mathcal{L}=\sum_{n} i \bar{\psi}_{n} \gamma_{\mu}\left(\partial_{\mu}-i \frac{e}{\pi_{c L}} \Phi \delta_{\mu, x}\right) \psi_{n},
$$

where $n$ runs over the $L d\left(\mathcal{k}_{F} \delta_{1}\right)$ values, $\partial x \rightarrow$ $\rightarrow 1 / R \partial_{\theta}, v_{F} \rightarrow v_{F}\left(\bar{c} / c_{0}\right)$.

The phases in all the $N_{\Phi}$ chains that are connected to the CD (17) are correlated provided $L \ll l_{y}, d \ll l_{d}$. This means that all the dual fermions located in these chains have the same transmission probability $g(\varepsilon)(10)$, in which $k$ [Eq. (11)] is replaced by $k\left(c_{0} / \bar{c}\right)$. Thus, the flux-dependent transport current carried by the bunch of $N_{\Phi}$ chains is nothing but $N_{\Phi} j_{q}(\Phi)$ where $j_{q}(\Phi)$ is given by Eq. (7). The parameter of transparency $\eta$ can be estimated quasiclassically:

$$
\eta \sim \exp \left(-\frac{\delta_{2}\left(m_{C D W} \Delta\right)^{1 / 2}}{\hbar}\right),
$$

where $m_{C D W}=\alpha^{-2} m^{*}, m^{*}$ is the band mass in $\mathrm{NbSe}_{3}$. Taking $m^{*}=1.8 m_{0}, \xi_{0}=30 \AA, \Delta=350 \mathrm{~K}$, $\alpha \approx 0.5$ (see, e.g., Grüner [2]), we get:

$$
\eta \approx 0.03-0.05
$$

for $\delta_{2} / \xi_{0} \approx 6-7[12,13]$. Note, however, that the ratio $\delta_{2} / \xi_{0}$ was estimated for a point defect. For a $\mathrm{CD}$, the estimate can differ though not crucially. 


\section{Calculation of a flux-dependent transport current}

The total current $I^{C D W}$ is the sum over the single chain currents. At small concentration of CDs [1] one can neglect the contribution of chains connecting the different loops (random loop approximation) we then get

$$
I^{C D W} \approx N j_{c}+\sum_{\text {loops }} j_{q}(\Phi)=I_{0}^{C D W}+I^{C D W}(\Phi),(20)
$$

where $N$ is the total number of chains in a sample, $I_{c}^{C D W}=N j_{C}$.

The sum over loops is estimated as

$$
\sum_{\text {loops }} \approx N_{\Phi} N_{C D}
$$

where $N_{C D}$ is the total number of loops (of CDs) in a sample:

$$
N_{C D}=n_{d} L_{\|} L_{\perp},
$$

where $n_{d}$ is the concentration of CDs, $L_{\|}$is the distance between the electrodes along the $x$-direction, $L_{\perp}$ is the size of a sample in the $y$-direction. Collecting together Eqs. (7), (20), (21) we obtain

$I^{C D W}(\Phi)=\frac{L d}{\pi} n_{2} n_{d} L_{\|} L_{\perp} j_{q}(\Phi)=\frac{L L_{\|}}{2 \pi} n_{d} I_{0}^{C D W} \times$ $\times \frac{1}{\tilde{p}_{F} v_{D}} \int d \varepsilon g(\varepsilon)\left[f_{0}\left(\varepsilon+\tilde{p}_{F} v_{D}\right)-f_{0}\left(\varepsilon-\tilde{p}_{F} v_{D}\right)\right]$.

To calculate the integral over the energy, we make use of the periodicity of $g(\varepsilon)$ :

$$
g(\varepsilon)=g\left(\varepsilon+2 \pi T_{0}\right),
$$

where

$$
k_{B} T_{0}=\frac{\hbar c}{L}
$$

is the interlevel spacing in a one-dimensional isolated ring. We get:

$$
\begin{gathered}
\frac{1}{\tilde{p}_{F} v_{D}} \int d \varepsilon g(\varepsilon)\left[f_{0}\left(\varepsilon+\tilde{p}_{F} v_{D}\right)-f_{0}\left(\varepsilon-\tilde{p}_{F} v_{D}\right)\right]= \\
2 \pi T_{0} \\
=\frac{1}{\tilde{p}_{F} v_{D}} \int_{0} d \varepsilon g\left(\varepsilon+\tilde{\varepsilon}_{F}\right) \sum_{n=-\infty}^{\infty}\left[f_{0}\left(\varepsilon+\tilde{\varepsilon}_{F}+2 \pi n T_{0}+\tilde{p}_{F} v_{D}\right)-f_{0}\left(\varepsilon+\tilde{\varepsilon}_{F}+2 \pi n T_{0}-\tilde{p}_{F} v_{D}\right)\right],
\end{gathered}
$$

where $\tilde{\varepsilon}_{F}$ is the Fermi energy for dual fermions.

Making use of the Poisson summation formula

$$
\sum_{n} p(n)=\int d n p(n)+2 \sum_{k=1}^{\infty} \int d n p(n) \cos (2 \pi k n)
$$

we get

$$
\sum_{n=-\infty}^{\infty}\left[f_{0}\left(\varepsilon+\tilde{\varepsilon}_{F}+2 \pi n T_{0}+\tilde{p}_{F} v_{D}\right)-f_{0}\left(\varepsilon+\tilde{\varepsilon}_{F}+2 \pi n T_{0}-\tilde{p}_{F} v_{D}\right)\right]=\frac{T}{T_{0}} \sum_{k=1}^{\infty} \frac{\sin \left(k \tilde{p}_{F} v_{D} / T_{0}\right) \sin \left(k \varepsilon / T_{0}\right)}{\operatorname{sh}\left(\pi k T / T_{0}\right)}
$$

Making use of the inequality $v_{D}<\bar{c}$, we eventually obtain the following equation for $I^{C D W}(\Phi)$ :

$$
I^{C D W}(\Phi)=\frac{L L_{\|} n_{d}}{2 \pi} \frac{T}{T_{0}} I_{0}^{C D W} \sum_{k=1}^{\infty} k \operatorname{sh}^{-1}\left(\pi k T / T_{0}\right) F\left(\Phi, \eta, T_{0}\right)
$$


where

$$
F\left(\Phi, \eta, T_{0}\right)=\int_{0}^{2 \pi T_{0}} d \varepsilon \sin \left(k \varepsilon / T_{0}\right) g\left(\varepsilon+\tilde{\varepsilon}_{F}\right) .
$$

In an actual experiment [2], the ratio $\pi T / T_{0} \approx 10$ which allows us to keep only the first harmonics with $k=1$ in the r.h.s. of Eq. (29). The exponential decrease with temperature is a typical feature of the AB-transport current (see, e.g., [14]), as well as of the persistent current [4].

The integral over the energy (30) has to be calculated at $\eta<<1$ [see Eq. (19a)]:

$$
F \approx 4 \eta^{2} \cos ^{2}\left(\pi \Phi / \Phi_{s}\right) \int_{0}^{2 \pi T_{0}} d \varepsilon \frac{\sin \left(\varepsilon / T_{0}\right) \sin ^{2}\left(\left(\varepsilon+\tilde{\varepsilon}_{F}\right) / 2 T_{0}\right)}{\eta^{2} \sin ^{2}\left(\left(\varepsilon+\tilde{\varepsilon}_{F}\right) / 2 T_{0}\right)+\left[\cos ^{2}\left(\pi \Phi / \Phi_{s}\right)-\eta-\cos \left(\left(\varepsilon+\tilde{\varepsilon}_{F}\right) / 2 T_{0}\right)\right]^{2}} .
$$

The main contribution to $F$ comes from those points in which the denominator of the integrand is close to zero. We get the asymptotes:

$$
\frac{1}{T_{0}} F \approx\left\{\begin{array}{l}
-\frac{1}{2} \eta \sin \left(\frac{\tilde{\varepsilon}_{F}}{T_{0}}\right)\left(1+\pi^{2}\left(\frac{\Phi-n \Phi_{s}}{\Phi_{s}}\right)^{2}\right), \quad \text { when }\left|\Phi-n \Phi_{s}\right|<\left\langle\Phi_{s}, \quad\left|\Phi-(n \pm 1 / 4) \Phi_{s}\right|>>\eta \Phi_{s},\right. \\
\frac{1}{4} \eta \cos \left(\frac{\tilde{\varepsilon}_{F}}{T_{0}}\right)\left(1 \mp 2 \pi\left(\frac{\Phi-(n \pm 1 / 4) \Phi_{s}}{\Phi_{s}}\right)\right) \text {, when }\left|\Phi-n \Phi_{s}\right|>>\eta \Phi_{s}, \quad\left|\Phi-(n \pm 1 / 4) \Phi_{s}\right|<\eta \Phi_{s}, \\
\frac{1}{\sqrt{2}} \eta^{3 / 2} \sin \left(\frac{\tilde{\varepsilon}_{F}}{T_{0}}\right) 2 \pi^{2}\left(\frac{\Phi-(n \pm 1 / 2) \Phi_{s}}{\Phi_{s}}\right)^{2}, \text { when }\left|\Phi-(n \pm 1 / 2) \Phi_{s}\right|<<\eta \Phi_{s}, \quad\left|\Phi-n \Phi_{s}\right|>>\Phi_{s} .
\end{array}\right.
$$

Here $n=\left[\Phi / \Phi_{s}\right]$ is an integer.

In an actual experiment [2], $n_{d} L_{\|} L \approx 10^{3}-10^{4}$ and we obtain the following estimate for the relative magnitude of the oscillatory component of $I^{C D W}$ :

$$
\frac{I^{C D W}(\Phi)}{I_{0}^{C D W}} \approx 10^{-2}-10^{-3}
$$

which is in good agreement with the experimental data [2].

To conclude, we have constructed, for the first time, a microscopic theory of the AB-oscillations phenomenon in a transport CDW-current which flows through a sample containing a large number of identical tiny holes (CD) placed in a strong magnetic field. The quantum CDW excitations view these holes as elementary solenoids which carry magnetic flux and the quantum transport current acquires terms oscillating with the magnetic flux with the period $h c / 2 e$. The oscillatory compo- nent of current is proportional to the concentration of CDs. Because of the long-range CDW rigidity, all the holes contribute additively to the current and the oscillations exist at sufficiently high temperature $T \approx 50 \mathrm{~K}$. At such temperatures, the single electron oscillations, periodic with $h c / e$, are exponentially suppressed by the electron-phonon phasebreaking scattering and cannot be observed.

The presented theory of the $\mathrm{ABE}$ in $\mathrm{CDW}$ is based on the quantum description of the CDW charge carriers in one dimension by means of the Bose-Fermi equivalence procedure. This method, together with the idea of a long range CDW coherence within an array of $1 D$ chains, allows us to reduce this $3 D$ problem to an exactly solvable model of a single chain connected to a loop pierced by a magnetic flux. This simple picture gives good agreement with the experimental data. 
I acknowledge discussions with I. Krive, A. Kovalev, Yu. Latyshev, and P. Monceau. I acknowledge Yurij Pershin for technical assistance. This work was supported by the Bilateral Collaboration Program between the CRTBT (Grenoble) and ILTPE (Kharkov).

1. For a review see Electronic Properties of Inorganic Quasi One-Dimensional Compounds, P. Monceau (ed.), Reidel Company, Dordrecht (1985), Parts 1 and 2; Charge Density Waves in Solids, L. Gork'ov and G. Grüner (eds.), Modern problems in Condensed Matter Sciences 25, NorthHolland, Amsterdam (1989); Electronic Crystals, ECRYS 93, S. Brazovskii and P. Monceau (eds.), J. Phys. (France) IV Coloq. C2, (1993); Density Waves in Solids, G. Grüner (ed.), Addison-Wesley, Reading, Massachusets (1994); I. V. Krive, A. S. Rozhavsky, and I. O. Kulik, Fiz. Nizk. Temp. 12, 1123 (1986) [Sov. J. Low Temp. Phys. 12, 635 (1986)]

2. Yu. I. Latyshev, O. Laborde, P. Monceau, and S. Klaumünzer, Phys. Rev. Lett. 78, 919 (1997).

3. I. V. Krive and A. S. Rozhavsky, in: Topological Phases in Quantum Theory, World Scientific, Singapore (1989) p. 291; Proc. Int. Seminar on «Geometric Aspects of Quantum Theory», Dubna, USSR (1989).

4. E. N. Bogachek, I. V. Krive, I. O. Kulik, and A. S. Rozhavsky, Phys. Rev. B42, 7614 (1990); E. N. Bogachek, I. V. Krive, I. O. Kulik, and A. S. Rozhavsky, Zh. Eksp. Teor. Fiz. 97, 603 (1990) [Sov. Phys. JETP 70, 336
(1990)]; E. N. Bogachek, I. V. Krive, and A. S Rozhavsky, Teor. Mat. Fiz. 83, 115 (1990) [Sov. Theor. Mat. Phys. 83, 1 (1990)]; I. V. Krive and A. S. Rozhavsky, Int. J. Mod. Phys. B6, 1255 (1992).

5. A. S. Rozhavsky, Fiz. Nizk. Temp. 22, 462 (1996) [Low Temp. Phys. 22, 360 (1996)].

6. S. Coleman, Phys. Rev. D11, 2088 (1975); S. Mandelstam, Phys. Rev. D11, 3026 (1975).

7. I. V. Krive and A. S. Rozhavsky, Usp. Fiz. Nauk 152, 33 (1987) [Sov. Phys. Usp. 152, (1987)].

8. I. V. Krive and A. S. Rozhavsky, Phys. Lett. A113, 313 (1985); Z.-B. Su and B. Sakita, Phys. Rev. Lett. 56, 9 (1986).

9. M. Büttiker, Y. Imry, and M. Ya. Azbel, Phys. Rev. A30, 1982 (1984).

10. Y. Imry, in: Directions in Condensed Matter Physecs, G. Grinstein and G. Mazenko (eds.), World Scientific, Singapore (1986), p. 101

11. A. S. Kovalev, Yu. V. Pershin, and A. S. Rozhavsky, Phys. Rev. B53, 16227 (1996).

12. I. Tüttö and A. Zawadowski, Phys. Rev. B32, 2449 (1985); J. R. Tucker, Phys. Rev. B40, 5447 (1989); P. A. Lee and T. M. Rice, Phys. Rev. B19, 3970 (1979).

13. S. Abe, J. Phys. Soc. Jpn. 54, 3494 (1985); M. Inui, P. P. Hall, S. Doniach, and A. Zettl, Phys. Rev. B38, 13047 (1988); C. M. Marcus, S. H. Strogatz, and R. M. Westervelt, Phys. Rev. B40, 5588 (1989).

14. M. R. Geller, D. Loss, and G. Kirczenow, Phys. Rev. Lett. 77, 5110 (1996). 